\title{
A Digital Index Theorem
}

\author{
R. Ayala ${ }^{1}$, E. Domínguez ${ }^{2}$, A.R. Francés ${ }^{2}$, A. Quintero $^{1}$ \\ 1 Dpt. de Geometría y Topología. Facultad de Matemáticas. Universidad de Sevilla. \\ Apto. 1160. E-41080 - Sevilla. Spain. \\ e-mail: quintero@cica.es \\ 2 Dpt. de Informática e Ingeniería de Sistemas. Facultad de Ciencias. \\ Universidad de Zaragoza. E-50009 - Zaragoza. Spain. \\ e-mail: afrances@posta.unizar.es
}

\begin{abstract}
This paper is devoted to prove a Digital Index Theorem for digital $(n-1)$-manifolds in a digital space $\left(R^{n}, f\right)$, where $f$ belongs to a large family of lighting functions on the standard cubical decomposition $R^{n}$ of the $n$-dimensional Euclidean space. As an immediate consequence we obtain the corresponding theorems for all $(\alpha, \beta)$-surfaces of Kong-Roscoe, with $\alpha, \beta \in\{6,18,26\}$ and $(\alpha, \beta) \neq$ $(6,6),(18,26),(26,26)$, as well as for the strong 26-surfaces of Bertrand-Malgouyres.
\end{abstract}

Key words: Index Theorem, weak lighting functions, digital $n$-manifold.

\section{Introduction}

In [1], [5] we introduce a general notion of digital manifold which includes the $(\alpha, \beta)$ surfaces of Kong-Roscoe [9] and the strong 26-surfaces of Bertrand-Malgouyres [6] as particular cases. In the same paper [5] we give a proof of the Digital Jordan-Brouwer Separation Theorem for a digital $(n-1)$-manifold $M$ in a digital space $(K, f)$ for which the continuous analogue is the $n$-dimensional Euclidean space $\mathbb{R}^{n}$. Although the Digital Jordan-Brouwer Theorem is an important result on its own, from the viewpoint of applications a digital counterpart of the topological index theorem is needed to characterize the digital components of the complement of $M$.

For dimension 3, Morgenthaler-Rosenfeld [13] gave a Digital Index Theorem for $(26,6)$ and $(6,26)$-surfaces in $\mathbb{Z}^{3}$. Later in [2], we used an early version of our multilevel architecture to genelarize the Morgenthaler-Rosenfeld result for $(26,6)$-surfaces to arbitrary closed hypersurfaces in a digital space $\left(R^{n}, f\right)$ for a special weak lighting function $f$ defining the $\left(3^{n}-1,2 n\right)$-adjacency in $\mathbb{Z}^{n}$.

In this paper we extend the Digital Index Theorem in [2] to digital spaces $\left(R^{n}, f\right)$ for which $f$ belongs to a quite large family of lighting functions. Doing that, we obtain as immediate consequences the Digital Index Theorem of Morgenthaler-Rosenfeld for both $(26,6)$ - and $(6,26)$-surfaces as part of a common theorem for all $(\alpha, \beta)$-surfaces, with $\alpha, \beta \in\{6,18,26\}$ and $(\alpha, \beta) \neq(6,6),(18,26),(26,26)$, as well as a Digital Index Theorem for the strong 26-surfaces of Bertrand-Malgouyres. At this point we must say that Malgouyres [12 has proved a Digital Index Theorem for certain class of digital surfaces which have not been yet studied within our framework. 


\section{Preliminaries}

In this section we follow [5] to introduce the framework in which we state and prove the Digital Index Theorem. This framework has evolved along a series of papers [1]-[4], the ultimate of which is [5]. Our approach to digital topology is based on a multilevel architecture that describes the discrete nature of digital images and provides a "continuous interpretation" for them as well.

The first level of this architecture is called the device model. It is an abstraction of the spatial layout of pixels in a digital image, which are represented by the $n$-cells of a homogeneously $n$-dimensional locally finite polyhedral complex $K$. Namely, $K$ is a complex of convex cells (polytopes) such that each cell is face of a finite number (nonzero) of $n$-cells. In this way, the objects displayed in digital images are (discrete) subsets of the set $\operatorname{cell}_{n}(K)$ of $n$-cells in a device model $K$; and, thus, we call a digital object in $K$ to any subset $O \subseteq \operatorname{cell}_{n}(K)$. However, when one looks at a digital image, these discrete objects are usually perceived as continuous objects. We will represent this fact, the objects' continuous perception, associating every digital object with a polyhedron called its continuous analogue. But, depending on the application, a given digital object may be perceived in several different continuous ways. A weak lighting function $f$ on a device model $K$ is a selection map that, subject to five quite natural conditions, chooses a continuous interpretation (i.e., a continuous analogue) for each digital object in $K$; and so, we call a digital space to any pair $(K, f)$. In order to recall the notion of weak lighting function we need the following notation.

Let $K$ be a device model and $\gamma, \sigma$ cells in $K$. We shall write $\gamma \leq \sigma$ if $\gamma$ is a face of $\sigma$, and $\gamma<\sigma$ if in addition $\gamma \neq \sigma$. If $|K|$ denotes the underlying polyhedron of $K$, a centroid-map is a map $c: K \rightarrow|K|$ such that $c(\sigma)$ belongs to the interior (as a cell) of $\sigma$; that is, $c(\sigma) \in \sigma-\partial \sigma$, where $\partial \sigma=\cup\{\gamma ; \gamma<\sigma\}$ stands for the boundary of $\sigma$. Given a cell $\alpha \in K$ and a digital object $O \subseteq \operatorname{cell}_{n}(K)$, the star of $\alpha$ in $O$ and the extended star of $\alpha$ in $O$ are respectively the digital objects $\operatorname{st}_{n}(\alpha ; O)=\{\sigma \in O ; \alpha \leq \sigma\}$ and $\operatorname{st}_{n}^{*}(\alpha ; O)=\{\sigma \in O ; \alpha \cap \sigma \neq \emptyset\}$. Notice that if $\operatorname{dim} \alpha=0$ then $\operatorname{st}_{n}(\alpha ; O)=$ $\operatorname{st}_{n}^{*}(\alpha ; O)$ for any digital object $O$ in $K$. The support of $O, \operatorname{supp}(O)$, is the set of all cells $\alpha \in K$ such that $\alpha=\cap\left\{\sigma ; \sigma \in\right.$ st $\left._{n}(\alpha ; O)\right\}$. To ease the writing, we shall use the following notation: $\operatorname{supp}(K)=\operatorname{supp}\left(\operatorname{cell}_{n}(K)\right)$, $\operatorname{st}_{n}(\alpha ; K)=\operatorname{st}_{n}\left(\alpha ; \operatorname{cell}_{n}(K)\right)$ and $\mathrm{st}_{n}^{*}(\alpha ; K)=\mathrm{st}_{n}^{*}\left(\alpha ; \operatorname{cell}_{n}(K)\right)$. Finally, we shall write $\mathcal{P}(A)$ for the family of all subsets of a given set $A$.

Given a device model $K$, a function $f: \mathcal{P}\left(\operatorname{cell}_{n}(K)\right) \times K \rightarrow\{0,1\}$ is said to be a weak lighting function (w.l.f.) on $K$ if it verifies the following five properties for all $O \in \mathcal{P}\left(\operatorname{cell}_{n}(K)\right)$ and $\alpha \in K$.

1. If $\alpha \in O$ then $f(O, \alpha)=1$.

2. If $\alpha \notin \operatorname{supp}(O)$ then $f(O, \alpha)=0$.

3. $f(O, \alpha) \leq f\left(\operatorname{cell}_{n}(K), \alpha\right)$.

4. $f(O, \alpha)=f\left(\operatorname{st}_{n}^{*}(\alpha ; O), \alpha\right)$.

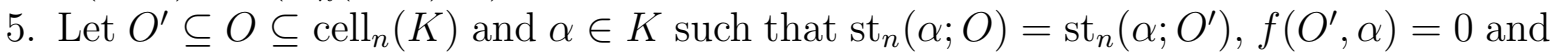
$f(O, \alpha)=1$. Then, the set of cells $\alpha\left(O^{\prime} ; O\right)=\left\{\beta<\alpha ; f\left(O^{\prime}, \beta\right)=0, f(O, \beta)=1\right\}$ is not empty and connected in $\partial \alpha$. Moreover, if any digital object $\bar{O}$ is such that $O \subseteq \bar{O}$, then $f(\bar{O}, \beta)=1$ for every $\beta \in \alpha\left(O^{\prime} ; O\right)$.

If $f(O, \alpha)=1$ we say that the w.l.f. $f$ lights the cell $\alpha$ for the digital object $O$, and 
we shall consider that $\{\alpha \in K ; f(O, \alpha)=1\}$ is the set of cells perceived when one looks at $O$ under the continuous interpretation provided by $f$.

Using this identification, the intuitive ideas underlying properties (1)-(5) above are the following. Property (1) expresses that, under any continuous interpretation, the pixels ( $n$-cells) in a digital object $O$ are always perceived. In addition to these pixels, we can only perceive cells in the support of $O, \operatorname{supp}(O)$, by property (2). Actually, the lighting function determines what lower dimensional cells in $\operatorname{supp}(O)$ are perceived. This is reasonable since the cells which are not in $\operatorname{supp}(O)$ lie in the border of the object and, moreover, they provide not information about how the pixels in $O$ are linked to each other. Properties (3) and (4) ensure that the perception of different objects is compatible. The first one states that a cell $\alpha$ is lighted for the digital object $\operatorname{cell}_{n}(K)$, consisting of all the pixels in the space, whenever it is lighted for some smaller object $O$; while the second says that whether a cell $\alpha$ is lighted or not for a given object $O$ is a local property of the object, and so it depends on the "neighbourhood" of $\alpha$ in $O$ given by the set $\operatorname{st}_{n}^{*}(\alpha ; O)$. Finally, property (5) is needed to avoid certain paradoxes related to the connectivity of the complement of objects (see Example 3.5 in [5]).

Although the continuous interpretation of every digital object in a digital space $(K, f)$ is provided by the w.l.f. $f$, to handle more easily with this information we associate a polyhedron $\left|\mathcal{A}_{O}^{f}\right|$ with each digital object $O$. As quoted above, $\left|\mathcal{A}_{O}^{f}\right|$ is called the continuous analogue of $O$, and it is derived from $f$ through other intermediate levels of the object $O$ as follows.

The device level of $O$ is the subcomplex $K(O)=\{\alpha \in K ; \alpha \leq \sigma, \sigma \in O\}$ induced in the device model $K$ by the pixels in $O$. Notice that $K(O)$ can be considered as a device model itself, and so it describes the discrete structure of the object. Moreover, the map $f_{O}$ given by $f_{O}\left(O^{\prime}, \alpha\right)=f(O, \alpha) f\left(O^{\prime}, \alpha\right)$, for all $O^{\prime} \subseteq O$ and $\alpha \in K(O)$, is a w.l.f. on $K(O)$, and we call the pair $\left(K(O), f_{O}\right)$ the digital subspace of $(K, f)$ induced by $O$.

The logical level of $O$ is an undirected graph, $\mathcal{L}_{O}^{f}$, whose vertices are the centroids of pixels ( $n$-cells) in $O$ and two of them $c(\sigma), c(\tau)$ are adjacent if there exists a common face $\alpha \leq \sigma \cap \tau$ such that $f(O, \alpha)=1$. Notice that such a cell $\alpha$ is linking the pixel $\sigma$ to $\tau$ in $K$ and, since $\alpha$ is also lighted for the digital object $O$, we will consider that those pixels $\sigma$ and $\tau$ are connected in $O$. So, the logical level is the simplest representation of the connectivity of an object (see Theorem 2.2 below).

However, the graph $\mathcal{L}_{O}^{f}$ does not completely represent the continuous perception of $O$, as we cannot read in it how many lower dimensional cells of $K$ are connecting two given pixels. This information is provided in the conceptual level of $O$, which is a directed graph, $\mathcal{C}_{O}^{f}$, whose vertices are the centroids $c(\alpha)$ of all cells $\alpha \in K$ with $f(O, \alpha)=1$, and its directed edges are $(c(\alpha), c(\beta))$ with $\alpha<\beta$.

The order complex $\mathcal{A}_{O}^{f}$ associated to the digraph $\mathcal{C}_{O}^{f}$ is called the simplicial analogue of $O$. That is, $\left\langle x_{0}, x_{1}, \ldots, x_{m}\right\rangle$ is an $m$-simplex of $\mathcal{A}_{O}^{f}$ if $x_{0}, x_{1}, \ldots, x_{m}$ is a directed path in $\mathcal{C}_{O}^{f}$. This simplicial complex defines the simplicial level for the object $O$. And, finally, the continuous analogue of $O$ is defined as the underlying polyhedron $\left|\mathcal{A}_{O}^{f}\right|$ of $\mathcal{A}_{O}^{f}$.

Examples 2.1 (1) In this paper we will deal with the device model $R^{n}$, called the standard cubical decomposition of the $n$-dimensional Euclidean space $\mathbb{R}^{n}$. This device model $R^{n}$ is the complex determined by the collection of unit $n$-cubes in $\mathbb{R}^{n}$ whose edges are parallel to the coordinate axes and whose centers are in the set $\mathbb{Z}^{n}$. The centroid-map we will consider in $R^{n}$ associates to each cube $\sigma$ its barycenter $c(\sigma)$. In particular, if $\operatorname{dim} \sigma=n$ 


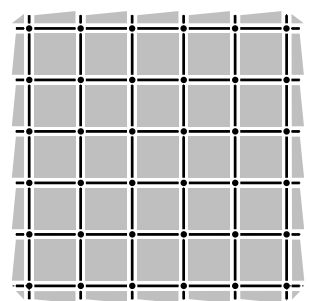

(a)

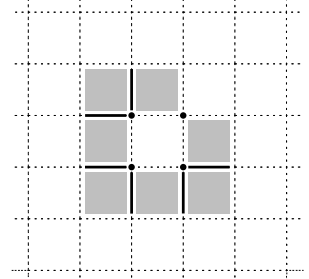

(b)

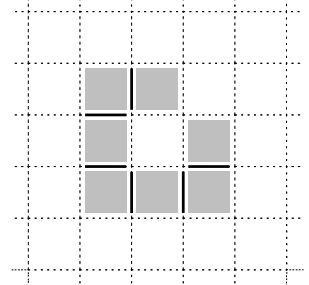

(c)

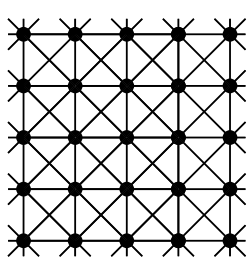

$\mathcal{L}_{R^{2}}^{f_{\max }}=\mathcal{L}_{R^{2}}^{g}$

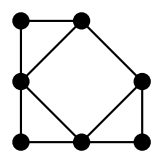

$\mathcal{L}_{O}^{f_{\max }}$

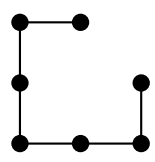

$\mathcal{L}_{O}^{g}$

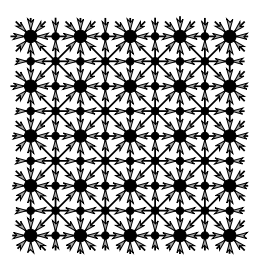

$\mathcal{C}_{R^{2}}^{f_{\max }}=\mathcal{C}_{R^{2}}^{g}$

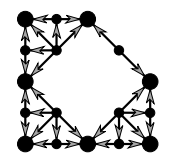

$\mathcal{C}_{O}^{f_{\max }}$

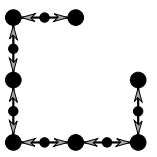

$\mathcal{C}_{O}^{g}$

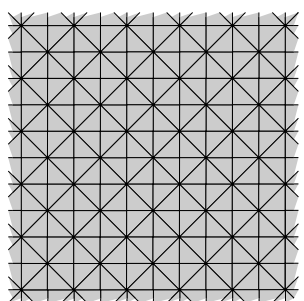

$\mathcal{A}_{R^{2}}^{f_{\max }}=\mathcal{A}_{R^{2}}^{g}$

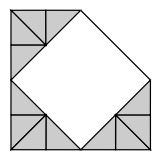

$\mathcal{A}_{O}^{f_{\max }}$

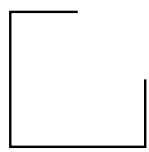

$\mathcal{A}_{O}^{g}$

Fig. 1. Levels of the objects $O$ and $\operatorname{cell}_{2}\left(R^{2}\right)$ for the w.l.f.'s $f_{\max }$ and $g$ in Example 2.1(2).

then $c(\sigma) \in \mathbb{Z}^{n}$. So that, every digital object $O$ in $R^{n}$ can be identified with a subset of points in $\mathbb{Z}^{n}$. Henceforth we shall use this identification without further comment.

(2) Every device model $K \neq \emptyset$ admits the weak lighting functions $f_{\max }$ and $g$ given respectively by:

(a) $f_{\max }(O, \alpha)=1$ if and only if $\alpha \in \operatorname{supp}(O)$

(b) $g(O, \alpha)=1$ if and only if $\alpha \in \operatorname{supp}(O)$ and $\operatorname{st}_{n}(\alpha ; K) \subseteq O$

In Fig. 1 are shown two objects, $O$ and $\operatorname{cell}_{2}\left(R^{2}\right)$, in the device model $R^{2}$, and their levels for these lighting functions. More precisely, Figs. 1(b) and 1(c) show the sets $\left\{\alpha \in R^{2} ; f_{\max }(O, \alpha)=1\right\}$ and $\left\{\alpha \in R^{2} ; g(O, \alpha)=1\right\}$ of cells that the w.l.f. $f_{\max }$ and $g$ light, respectively, for the object $O$. As these sets do not agree, all the levels of $O$ in the digital spaces $\left(R^{2}, f_{\max }\right)$ and $\left(R^{2}, g\right)$ are distinct, in particular $\left|\mathcal{A}_{O}^{f_{\max }}\right| \neq\left|\mathcal{A}_{O}^{g}\right|$. On the other hand, $\left\{\alpha \in R^{2} ; f_{\max }\left(\operatorname{cell}_{2}\left(R^{2}\right), \alpha\right)=1\right\}=\left\{\alpha \in R^{2} ; g\left(\operatorname{cell}_{2}\left(R^{2}\right), \alpha\right)=1\right\}$ (see Fig. 1(a)), and so all the levels of the object $\operatorname{cell}_{2}\left(R^{2}\right)$ are the same in these digital spaces.

For the sake of simplicity, we will usually drop " $f$ " from the notation of the levels of an object. Moreover, for the whole object $\operatorname{cell}_{n}(K)$ we will simply write $\mathcal{L}_{K}, \mathcal{C}_{K}$ and $\mathcal{A}_{K}$ for its levels. Notice that $\mathcal{A}_{K}$ is always a full subcomplex of the derived subdivision $K^{(1)}$ induced in the device model $K$ by the centroid-map $c$; and, for any digital object $O \subseteq \operatorname{cell}_{n}(K)$, its simplicial analogue $\mathcal{A}_{O}$ is also a full subcomplex of the simplicial analogue $\mathcal{A}_{K}$ of the whole digital space $(K, f)$; see Fig. 1 .

Next we recall the notion of connectedness given in [3]. Let $O$ and $O^{\prime}$ be two disjoint digital objects in a digital space $(K, f)$. Two distinct $n$-cells $\sigma, \tau \in O$ are said to be 
$O^{\prime}$-adjacent in $O$ if there exists a common face $\alpha \leq \sigma \cap \tau$ such that $f\left(O^{\prime}, \alpha\right)=0$ and $f\left(O \cup O^{\prime}, \alpha\right)=1$. An $O^{\prime}$-path in $O$ from $\sigma$ to $\tau$ is a finite sequence $\left(\sigma_{i}\right)_{i=0}^{m} \subseteq O$ such that $\sigma_{0}=\sigma, \sigma_{m}=\tau$ and $\sigma_{i-1}$ is $O^{\prime}$-adjacent in $O$ to $\sigma_{i}$, for $i=1, \ldots, m$. Then, a digital object $O$ will be said $O^{\prime}$-connected if for any pair of $n$-cells $\sigma, \tau \in O$ there exists an $O^{\prime}$-path in $O$ from $\sigma$ to $\tau$. And finally, an object $C \subseteq O$ is an $O^{\prime}$-component of $O$ if for any pair of $n$-cells $\sigma, \tau \in C$ there exists an $O^{\prime}$-path in $O$ from $\sigma$ to $\tau$ and none element in $C$ is $O^{\prime}$-adjacent in $O$ to some element of $O-C$. It can be proved that any $O^{\prime}$-component is an $O^{\prime}$-connected object itself (see [5]).

Given a digital object $O$ in the digital space $(K, f)$ the previous definitions provide an entire family of notions of connectedness for $O$ in relation to another object $O^{\prime}$, when $O^{\prime}$ is allowed to range over the set of all subsets of $\operatorname{cell}_{n}(K)-O$. The extreme cases, when $O^{\prime}=\emptyset$ and $O^{\prime}=\operatorname{cell}_{n}(K)-O$, represent the connectedness of the digital object $O$ itself and the connectedness of $O$ as the complement of $\operatorname{cell}_{n}(K)-O$, respectively. Thus, we will simply say that an object $O$ is connected if it is $\emptyset$-connected.

Next result shows how these notions of connectedness are stated at each level of our architecture. Below, given two subcomplexes $L_{1}$ and $L_{2}$ of a simplicial complex $L$, the simplicial complement of $L_{2}$ in $L_{1}$ will be denoted by $L_{1} \backslash L_{2}=\left\{\alpha \in L_{1} ; \alpha \cap\left|L_{2}\right|=\emptyset\right\}$.

Theorem 2.2 Let $O$ and $O^{\prime}$ be two disjoint digital objects in a digital space. The family $\mathcal{F}$ of $O^{\prime}$-components of $O$ can be described in any of the following ways

1. Conceptual level: $\mathcal{F}=\left\{O_{G}\right\}$, where $O_{G}=\{\sigma \in O ; c(\sigma)$ is a vertex of $G\}$, and $G$ ranges over the family of components of the digraph $\mathcal{C}_{O \cup O^{\prime}} \backslash \mathcal{C}_{O^{\prime}}$.

2. Simplicial level: $\mathcal{F}=\left\{O_{A}\right\}$, where $O_{A}=\{\sigma \in O ; c(\sigma) \in A\}$, and $A$ ranges over the family of components of the simplicial complement $\mathcal{A}_{O \cup O^{\prime}} \backslash \mathcal{A}_{O^{\prime}}$.

3. Continuous level: $\mathcal{F}=\left\{O_{X}\right\}$, where $O_{X}=\{\sigma \in O ; c(\sigma) \in X\}$, and $X$ ranges over the family of components of the space $\left|\mathcal{A}_{O \cup O^{\prime}}\right|-\left|\mathcal{A}_{O^{\prime}}\right|$.

Moreover, the connected components $\left(\emptyset\right.$-components) of $O$ are $\mathcal{F}=\left\{O_{L}\right\}$, where $O_{L}=$ $\{\sigma \in O ; c(\sigma)$ is a vertex of $L\}$, and $L$ ranges over the components of the logical level $\mathcal{L}_{O}$.

Since the continuous analogue provides the "continuous interpretation" of digital objects, the following definition arises naturally in our architecture. A digital object $M$ in a digital space $(K, f)$ is said to be a digital manifold if its continuous analogue $\left|\mathcal{A}_{M}\right|$ is a combinatorial manifold without boundary. In [3] we show that Kong-Roscoe's $(\alpha, \beta)$ surfaces, for $\alpha, \beta \in\{6,18,26\}$ and $(\alpha, \beta) \neq(6,6)$, are digital 2-manifolds in suitable digital spaces $\left(R^{3}, f_{\alpha \beta}\right)$; and in [4], [5] the strong 26-surfaces defined in $\mathbb{Z}^{3}$ by BertrandMalgouyres are characterized as the digital 2-manifolds of the digital space $\left(R^{3}, f^{B M}\right)$.

In this way, a digital Jordan-Brouwer separation theorem holds for each of these surfaces (see [9] and [6]), which can be obtained as corollaries of the following general result; see [5] for its proof as well as for the proof of Theorem 2.2 above.

Theorem 2.3 (Digital Jordan-Brouwer Theorem) Let $(K, f)$ be a digital space for which $\left|\mathcal{A}_{K}\right|=\mathbb{R}^{m}$. Let $M$ be a connected digital $(m-1)$-manifold in $(K, f)$. Then the complement cell $l_{n}(K)-M$ is divided into two $M$-components. Moreover, if $M$ is finite then one of the $M$-components is also finite. 


\section{Main results}

In an early version of our architecture [2] we have already stated and proved a Digital Index Theorem. There, we define directly the digital index at the logical level and then we show that, by transferring it through the levels of the architecture, we get the usual topological index at the continuous level. The proof of the Digital Index Theorem in [2] does not work if $(K, f)=\left(R^{n}, f\right)$, in Theorem 2.3, is an arbitrary digital space based on the standard cubical decomposition of the Euclidean $n$-space; see Remark 4.8 below. However, it is still possible to proceed along the same ideas in case we are given enough digital half-lines parallel to the coordinate axes which meet the manifold "appropriately". For this we require a further condition on the digital spaces, which is actually satisfied by the lighting functions which provide the $(\alpha, \beta)$-surfaces and the strong 26 -surfaces as well.

Next we state this further condition and define precisely what we mean by "appropriate" intersection.

Definition 3.1 A digital space $\left(R^{n}, f\right)$ is said to be solid if $f(O, \alpha)=1$ for all $\alpha \in R^{n}$ and $O \subseteq \operatorname{cell}_{n}\left(R^{n}\right)$ satisfying $\operatorname{st}_{n}\left(\alpha ; R^{n}\right) \subseteq O$.

Remark 3.2 Since $\operatorname{st}_{n}(\alpha ; K) \subseteq \mathrm{st}_{n}^{*}(\alpha ; K)$ in any digital space $(K, f)$ it follows that, for any cell $\alpha \in R^{n}$ and any digital object $O \subseteq \operatorname{cell}_{n}\left(R^{n}\right)$ with $\operatorname{st}_{n}^{*}\left(\alpha ; R^{n}\right) \subseteq O, f(O, \alpha)=1$ in case $\left(R^{n}, f\right)$ is solid. In particular, $f\left(\operatorname{cell}_{n}\left(R^{n}\right), \alpha\right)=1$ for any cell $\alpha \in R^{n}$. Therefore, $\left|\mathcal{A}_{R^{n}}\right|=\mathbb{R}^{n}$, and so the Digital Jordan-Brouwer Theorem holds for solid digital spaces $\left(R^{n}, f\right)$.

Given a solid digital space $\left(R^{n}, f\right)$ we need two lemmas to check that digital halflines meet digital objects "appropriately". The first one is an immediate consequence of Definition 3.1 and property (2) in the definition of w.l.f.'s.

Lemma 3.3 Let $O$ be a digital object in a solid digital space $\left(R^{n}, f\right)$. For any $(n-1)$ dimensional cell $\alpha \in R^{n}, f(O, \alpha)=1$ if and only if $\alpha \in \operatorname{supp}(O)$; that is, the two $n$-cells containing $\alpha$ are in $O$.

Although we can prove the existence of enough half-lines for an arbitrary w.l.f. on $R^{n}$ we use here Lemma 3.3 to find easily such half-lines in a solid digital space $\left(R^{n}, f\right)$ as follows. Let $\sigma \in \operatorname{cell}_{n}\left(R^{n}\right)$ be an $n$-cell with centroid $c(\sigma)=\left(x_{1}, x_{2}, \ldots, x_{n}\right) \in \mathbb{Z}^{n}$. Then Lemma 3.3 shows that the continuous analogue of the object

$$
H_{\sigma}=\left\{\tau \in \operatorname{cell}_{n}\left(R^{n}\right) ; c(\tau)=\left(x_{1}, \ldots, x_{n-1}, \lambda\right) \in \mathbb{Z}^{n}, \lambda \geq x_{n}\right\}
$$

is the Euclidean half-line $\left|\mathcal{A}_{H_{\sigma}}\right|=\left\{\left(x_{1}, \ldots, x_{n-1}, \lambda\right) \in \mathbb{R}^{n} ; \lambda \geq x_{n}\right\}$. This leads us to call $H_{\sigma}$ a digital half-line starting at $\sigma$. Similarly, for $k \geq 0$, the object $S_{\sigma}^{k}=\left\{\sigma_{0}, \sigma_{1}, \ldots, \sigma_{k}\right\}$, with $c\left(\sigma_{i}\right)=\left(x_{1}, \ldots, x_{n-1}, x_{n}+i\right) \in \mathbb{Z}^{n}(0 \leq i \leq n)$, is termed a digital segment of length $k$ since $\left|\mathcal{A}_{S_{\sigma}^{k}}\right|$ is the closed segment in $\mathbb{R}^{n}$ from $\left(x_{1}, \ldots, x_{n}\right)$ to $\left(x_{1}, \ldots, x_{n-1}, x_{n}+k\right)$. We shall regard any digital point $\sigma$ as the digital segment $S_{\sigma}^{0}=\{\sigma\}$ of length 0 . Similarly, digital half-lines are defined for the rest of coordinates $x_{i}, 1 \leq i \leq n-1$.

For a segment $S=S_{\sigma}^{k}$ we call predecessor and successor of $S$ to the digital points $\pi_{S}$ and $\sigma_{S}$ determined by $c\left(\pi_{s}\right)=\left(x_{1}, \ldots, x_{n-1}, x_{n}-1\right)$ and $c\left(\sigma_{S}\right)=\left(x_{1}, \ldots, x_{n-1}, x_{n}+k+1\right)$, respectively. 

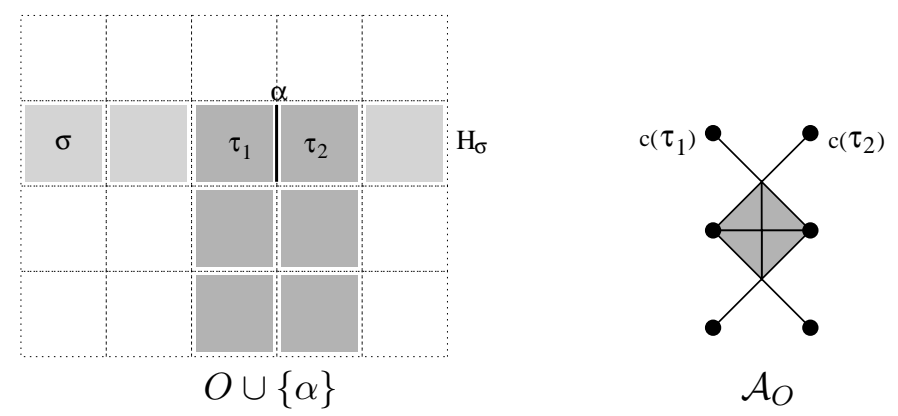

Fig. 2. In non-solid digital spaces, the intersection between digital half-lines and objects may be not "appropriate".

For an object $O$ and a half-line $H_{\sigma}$ in an arbitrary (i.e., not necessarily solid) digital space $\left(R^{n}, f\right)$ it is only possible to show the inclusion $\left|\mathcal{A}_{H_{\sigma}}\right| \cap\left|\mathcal{A}_{O}\right| \subseteq\left|\mathcal{A}_{H_{\sigma} \cap O}\right|$. Moreover, next example shows that, in general, this inclusion can be strict.

Example 3.4 Let $h$ be the w.l.f. defined on $R^{2}$ by $h(O, \alpha)=1$ if and only if: (a) $\operatorname{dim} \alpha=$ 2 and $\alpha \in O$; (b) $\operatorname{dim} \alpha=0$ and $\operatorname{st}_{2}\left(\alpha ; R^{2}\right) \subseteq O$; (c) $\operatorname{dim} \alpha=1$ and one of the two following conditions holds:

(c1) $\mathrm{st}_{2}^{*}(\alpha ; O)=\mathrm{st}_{2}^{*}\left(\alpha ; R^{2}\right)$

(c2) $\alpha \in \operatorname{supp}(O)$ and there exist $\sigma, \tau \in \mathrm{st}_{2}^{*}\left(\alpha ; R^{2}\right)-O$ such that $\sigma \cap \tau=\emptyset$.

Notice that the digital space $\left(R^{2}, h\right)$ is not solid since $\operatorname{st}_{2}\left(\alpha ; R^{2}\right) \subseteq O$, for the object $O$ and the 1-cell $\alpha$ depicted in Fig. 2, and however the definition of $h$ yields $h(O, \alpha)=0$. In any case, the object $H_{\sigma}$ in Fig. 2 is still a digital half-line; and the continuous analogue $\left|\mathcal{A}_{H_{\sigma} \cap O}\right|$ is the closed segment in $\mathbb{R}^{2}$ joining $c\left(\tau_{1}\right)$ and $c\left(\tau_{2}\right)$, where $\left\{\tau_{1}, \tau_{2}\right\}=\operatorname{st}_{2}\left(\alpha ; R^{2}\right)=$ $H_{\sigma} \cap O$, which contains strictly the set $\left\{c\left(\tau_{1}\right), c\left(\tau_{2}\right)\right\}=\left|\mathcal{A}_{H_{\sigma}}\right| \cap\left|\mathcal{A}_{O}\right|$.

Definition 3.5 Let $O$ be a digital object in $\left(R^{n}, f\right)$. We say that $H_{\sigma}$ meets $O$ appropriately if $\left|\mathcal{A}_{H_{\sigma}}\right| \cap\left|\mathcal{A}_{O}\right|=\left|\mathcal{A}_{H_{\sigma} \cap O}\right|$.

The following lemma, whose proof is straightforward from Lemma 3.3 , shows that in a solid digital space $\left(R^{n}, f\right)$ the digital half-lines which are parallel to the coordinate axes meet any digital object $O$ appropriately. We conjecture that appropriate intersections still occur in arbitrary digital spaces $\left(R^{n}, f\right)$ if the digital object $O$ is chosen to be a digital $(n-1)$-manifold. Of course, a positive answer to that conjecture would allow us to remove the hypothesis on the solidness of $\left(R^{n}, f\right)$ in Theorem 3.8 below.

Lemma 3.6 Let $H_{\sigma}$ be a digital half line in the solid digital space $\left(R^{n}, f\right)$. Then $\left|\mathcal{A}_{H_{\sigma}}\right| \cap$ $\left|\mathcal{A}_{O}\right|=\left|\mathcal{A}_{H_{\sigma} \cap O}\right|$ for any digital object $O \subseteq$ cell $_{n}\left(R^{n}\right)$. Moreover, the object $C$ is a component of $H_{\sigma} \cap O$ if and only if $\left|\mathcal{A}_{C}\right|$ is a component of $\left|\mathcal{A}_{H_{\sigma} \cap O}\right|$. In particular, if $O$ is finite, the components of $H_{\sigma} \cap O$ are digital segments. In addition, if $\sigma \notin O$ the predecessor $\pi_{C}$ and the successor $\sigma_{C}$ of any component $C$ of $H_{\sigma} \cap O$ are not in $O$.

Next we define the digital index function $i_{\operatorname{dig}}(-; M)$ for $n$-cells in the complement of a digital $(n-1)$-manifold $M$. For this we use the following notation. Let $O$ be a digital object in $\left(R^{n}, f\right)$. The cuboid of $O$ is the object $N(O)=\cup\left\{\operatorname{st}_{n}^{*}\left(\sigma ; R^{n}\right) ; \sigma \in O\right\}$; that is, $N(O)$ consists of all $n$-cells which meet $O$. 
Definition 3.7 Let $M$ be a digital $(n-1)$-manifold in a solid digital space $\left(R^{n}, f\right)$. Given $\sigma \in \operatorname{cell}_{n}\left(R^{n}\right)-M$ the digital index of $\sigma$ is the integer number $i_{\operatorname{dig}}(\sigma ; M)=\Sigma_{C} i_{\operatorname{dig}}(C ; M)$, where $C$ ranges over the set of components of $H_{\sigma} \cap M$ and $i_{\operatorname{dig}}(C ; M)$ is defined as follows. We write $i_{\operatorname{dig}}(C ; M)=0$, and we say that $H_{\sigma}$ meets $M$ tangentially at $C$, if both $\pi_{C}$ and $\sigma_{C}$ lie in the same $M$-component of $N(C)-M$. Otherwise we write $i_{\operatorname{dig}}(C ; M)=1$ and we say that $H_{\sigma}$ meets $M$ transversally at $C$.

We next state the following characterization of the points in the complement of a digital $(n-1)$-manifold in a solid digital space $\left(R^{n}, f\right)$.

Theorem 3.8 (Digital Index Theorem) Let $M$ be a finite digital $(n-1)$-manifold in a solid digital space $\left(R^{n}, f\right)$. A digital point $\sigma \in$ cell $_{n}\left(R^{n}\right)-M$ belongs to the finite $M$-component of the complement of $M$ if and only if $i_{\operatorname{dig}}(\sigma ; M)$ is odd.

As it was quoted above, the digital spaces $\left(R^{3}, f_{\alpha \beta}\right)$ and $\left(R^{3}, f^{B M}\right)$ which provide the $(\alpha, \beta)$-surfaces and the strong 26-surfaces, respectively, are solid. This fact is immediately checked from the definition of the corresponding lighting functions $f_{\alpha \beta}$ and $f^{B M}$. Moreover, in [3] it is shown that the w.l.f.'s $f_{\alpha \beta}$ provide the usual $(\alpha, \beta)$-connectedness on $R^{3}$ for objects and its complements; that is,

(a) $C$ is a connected component of the digital object $O$ in $\left(R^{3}, f_{\alpha \beta}\right)$ if and only if it is a $\alpha$-component of $O$; and,

(b) $C$ is an $O$-component of the complement $\operatorname{cell}_{3}\left(R^{3}\right)-O$ of $O$ in $\left(R^{3}, f_{\alpha \beta}\right)$ if and only if it is a $\beta$-component of $O$.

Similarly, the lighting function $f^{B M}$ provides the $(26,6)$-connectedness on $R^{3}$ (see [5]). So the following Digital Index Theorem hold for these surfaces as a consequence of Theorem 3.8 .

Corollary 3.9 Let $S$ be a finite $(\alpha, \beta)$-surface (strong 26-surface) in $\mathbb{Z}^{3}$, with $\alpha, \beta \in$ $\{6,18,26\}$ and $(\alpha, \beta) \neq(6,6),(18,26),(26,26)$. Then, a digital point $x \in \mathbb{Z}^{3}-S$ belongs to the finite $\beta$-component (26-component, respectively) of $\mathbb{Z}^{3}-S$ if and only if $i_{\operatorname{dig}}(x ; S)$ is odd.

Notice that, since all the $(18,26)$ - and $(26,26)$-surfaces are coordinate planes (see [9]), and so infinite surfaces, a Digital Index Theorem has no sense for them, even though the digital spaces $\left(R^{3}, f_{18,26}\right)$ and $\left(R^{3}, f_{26,26}\right)$ providing these surfaces are solid.

We prove our main result, Theorem 3.8, in the next section.

\section{Proof of Theorem 3.8}

Throughout this section we shall assume that $M$ is a finite digital $(n-1)$-manifold in a solid digital space $\left(R^{n}, f\right)$. The crucial point in the proof of Theorem 3.8 is Proposition 4.1 below which allows us to apply the Topological Index Theorem (see Appendix A). Given $\sigma \in \operatorname{cell}_{n}\left(R^{n}\right)-M$ let $H_{\sigma}$ denote the half-line starting at $\sigma$ as in Lemma 3.6. Recall that the cuboid of an object $O \subseteq R^{n}$ is $N(O)=\cup\left\{\mathrm{st}_{n}^{*}\left(\sigma ; R^{n}\right) ; \sigma \in O\right\}$.

Proposition 4.1 The pairs $\left(\left|\operatorname{st}\left(\mathcal{A}_{C} ; \mathcal{A}_{N(C)}\right)\right|,\left|\operatorname{st}\left(\mathcal{A}_{C} ; \mathcal{A}_{M}\right)\right|\right)$, where $C$ ranges over the set of components of $H_{\sigma} \cap M$, form an admissible family of relative balls for $\left|\mathcal{A}_{H_{\sigma} \cap M}\right|=$ $\left|\mathcal{A}_{H_{\sigma}}\right| \cap\left|\mathcal{A}_{M}\right|$; see Appendix $A$. 
In the proof of 4.1 we shall need the following lemmas

Lemma 4.2 Let $S=S_{\tau}^{k}$ be a digital segment. Then $\operatorname{st}\left(\mathcal{A}_{S} ; \mathcal{A}_{R^{n}}\right) \subseteq \mathcal{A}_{O}$ for all digital object $O \subseteq \operatorname{cell}_{n}\left(R^{n}\right)$ containing $N(S)$. In particular, st $\left(\mathcal{A}_{S} ; \mathcal{A}_{R^{n}}\right)=\operatorname{st}\left(\mathcal{A}_{S} ; \mathcal{A}_{O}\right)$.

Proof. As $\mathcal{A}_{O}$ is a full subcomplex of $\mathcal{A}_{R^{n}}$, it will suffice to show the inclusion for the sets of vertices. Moreover by Lemma 3.3 the inclusion $S \subseteq O$ yields $\mathcal{A}_{S} \subseteq \mathcal{A}_{O}$. Therefore we can argue with a vertex $c(\alpha) \in \operatorname{st}\left(\mathcal{A}_{S} ; \mathcal{A}_{R^{n}}\right)-\mathcal{A}_{S}$. For such a vertex there exists a 1-simplex $\langle c(\alpha), c(\rho)\rangle \in \mathcal{A}_{R^{n}}$ with $c(\rho) \in \mathcal{A}_{S}$; that is, $\rho$ is either an $n$-cube in $S$ or $\rho$ is the common $(n-1)$-face of two $n$-cubes in $S$. In any case $\alpha<\rho$, and so $\operatorname{st}_{n}^{*}\left(\alpha ; R^{n}\right) \subseteq$ st $_{n}^{*}\left(\rho ; R^{n}\right) \subseteq N(S) \subseteq O$; hence $c(\alpha) \in \mathcal{A}_{O}$ by Remark 3.2.

Corollary 4.3 For $O=N(S)$ we get $\operatorname{st}\left(\mathcal{A}_{S} ; \mathcal{A}_{N(S)}\right)=\operatorname{st}\left(\mathcal{A}_{S} ; \mathcal{A}_{R^{n}}\right)$.

Lemma 4.4 Let $S=S_{\tau}^{k}$ be a digital segment and let $O \subseteq$ cell $_{n}\left(R^{n}\right)$ be a digital object with $S \subseteq O$. Then $\operatorname{st}\left(\mathcal{A}_{S} ; \mathcal{A}_{O}\right)=\operatorname{st}\left(\mathcal{A}_{S} ; \mathcal{A}_{N(S)}\right) \cap \mathcal{A}_{O}$. Moreover, if $O$ is a digital r-manifold then $\operatorname{st}\left(\mathcal{A}_{S} ; \mathcal{A}_{O}\right)$ is a combinatorial $r$-ball with boundary

$$
\partial \operatorname{st}\left(\mathcal{A}_{S} ; \mathcal{A}_{O}\right)=\left\{\left\langle c\left(\mu_{1}\right), \ldots, c\left(\mu_{s}\right)\right\rangle \in \mathcal{A}_{O} ; \mu_{s}<\sigma \cap \sigma^{\prime}, \sigma \in S, \sigma^{\prime} \in O-S\right\}
$$

Proof. According to Lemma 3.3 the inclusion $S \subseteq O$ yields $\mathcal{A}_{S} \subseteq \mathcal{A}_{O}$. Then $\operatorname{st}\left(\mathcal{A}_{S} ; \mathcal{A}_{O}\right)=$ $\operatorname{st}\left(\mathcal{A}_{S} ; \mathcal{A}_{N(S)}\right) \cap \mathcal{A}_{O}$. Here we use Corollary 4.3 as well as the well known equality $\operatorname{st}\left(L ; J_{1} \cap\right.$ $\left.J_{2}\right)=\operatorname{st}\left(L ; J_{1}\right) \cap J_{2}$ for subcomplexes $L, J_{1}, J_{2} \subseteq J$ with $L \subseteq J_{1} \cap J_{2}$ and $J_{1}$ and $J_{2}$ full subcomplexes.

In order to show the second part we proceed inductively on the length $k$ of $S=S_{\tau}^{k}$ as follows. For $k=0, S=\{\tau\}$ is a digital point; so that, $\mathcal{A}_{S}=\{c(\tau)\}$ is the centroid of $\tau$. Hence $\operatorname{st}\left(\mathcal{A}_{S} ; \mathcal{A}_{O}\right)$ is an $r$-ball in the combinatorial $r$-manifold $\left|\mathcal{A}_{O}\right|$. Moreover $\partial \operatorname{st}\left(\mathcal{A}_{S} ; \mathcal{A}_{O}\right)=\left\{\left\langle c\left(\mu_{1}\right), \ldots, c\left(\mu_{s}\right)\right\rangle \in \mathcal{A}_{O} ; \mu_{s}<\tau\right\}$, and by property (2) in the definition of w.l.f.'s there exists at least an $n$-cube $\sigma \neq \tau$ in $O-S$ with $\mu_{s}<\sigma$.

Assume the result holds for segments of length $k^{\prime}<k$. Then, for the segment $S=$ $\left\{\tau_{0}, \tau_{1}, \ldots, \tau_{k}\right\}, \tau_{0}=\tau$, the $\operatorname{star} \operatorname{st}\left(\mathcal{A}_{S} ; \mathcal{A}_{O}\right)=B_{1} \cup B_{2}$ is the union of the $r$-balls $B_{1}=$ $\operatorname{st}\left(\mathcal{A}_{S_{1}} ; \mathcal{A}_{O}\right)$ and $B_{2}=\operatorname{st}\left(c\left(\tau_{k}\right) ; \mathcal{A}_{O}\right)$, where $S_{1}=S-\left\{\tau_{k}\right\}$. Moreover, $D=B_{1} \cap B_{2}=$ $\operatorname{st}\left(c\left(\sigma_{k}\right) ; \partial \operatorname{st}\left(c\left(\tau_{k}\right) ; \mathcal{A}_{O}\right)\right)$, with $\sigma_{k}=\tau_{k-1} \cap \tau_{k}$, is an $(r-1)$-ball in the $(r-1)$-sphere $\partial \operatorname{st}\left(c\left(\tau_{k}\right) ; \mathcal{A}_{O}\right)$. Hence $\operatorname{st}\left(\mathcal{A}_{S} ; \mathcal{A}_{O}\right)$ is an $r$-ball by 3.16 in [15].

The formula of the boundary also follows inductively since $\partial \operatorname{st}\left(\mathcal{A}_{S} ; \mathcal{A}_{O}\right)$ is the union

$$
\begin{gathered}
\overline{\partial B_{1}-D \cup \overline{\partial B_{2}-D}=} \\
\left\{\left\langle c\left(\mu_{1}\right), \ldots, c\left(\mu_{s}\right)\right\rangle \in \mathcal{A}_{O} ; \mu_{s}<\sigma \cap \sigma^{\prime}, \sigma \in S_{1}, \sigma^{\prime} \in O-S_{1}, \sigma^{\prime} \neq \tau_{k}\right\} \cup \\
\left\{\left\langle c\left(\mu_{1}\right), \ldots, c\left(\mu_{s}\right)\right\rangle \in \mathcal{A}_{O} ; \mu_{s}<\tau_{k}, \mu_{s} \neq \sigma_{k}\right\}= \\
\left\{\left\langle c\left(\mu_{1}\right), \ldots, c\left(\mu_{s}\right)\right\rangle \in \mathcal{A}_{O} ; \mu_{s}<\sigma \cap \sigma^{\prime}, \sigma \in S, \sigma^{\prime} \in O-S\right\}
\end{gathered}
$$

where $\overline{K-L}$ stands for the set of simplexes which are faces of some $\sigma \in K$ with $\sigma \notin L$.

As an immediate consequence of Lemma 4.4 and Corollary 4.3 we obtain for $O=R^{n}$

Corollary 4.5 For the segment $S=S_{\tau}^{k}=\left\{\tau_{0}, \tau_{1}, \ldots, \tau_{k}\right\}$ the polyhedron

$$
B=\left|\operatorname{st}\left(\mathcal{A}_{S} ; \mathcal{A}_{N(S)}\right)\right|=\cup\left\{\tau_{i} ; 0 \leq i \leq k\right\}
$$


is an n-ball with boundary

$$
\begin{aligned}
& \partial B=\left|\left\{\left\langle c\left(\mu_{1}\right), \ldots, c\left(\mu_{s}\right)\right\rangle \in \mathcal{A}_{R^{n}} ; \mu_{s}<\sigma \cap \sigma^{\prime}, \sigma \in S, \sigma^{\prime} \in \operatorname{cell}_{n}\left(R^{n}\right)-S\right\}\right|= \\
& \cup\left\{\mu \in R^{n} ; \mu<\sigma \cap \sigma^{\prime}, \sigma \in S, \sigma^{\prime} \in \operatorname{cell}_{n}\left(R^{n}\right)-S\right\}
\end{aligned}
$$

and hence int $(B)=B-\partial B=\left(\cup_{0 \leq i \leq k} \stackrel{\circ}{\tau}_{i}\right) \cup\left(\cup\left\{\stackrel{\circ}{\sigma}_{i} ; \sigma_{i}=\tau_{i-1} \cap \tau_{i}, 0 \leq i \leq k\right\}\right)$

We are now ready for the proof of Proposition 4.1.

Proof of Proposition 4.1. According to Lemma 3.6, the components of $\left|\mathcal{A}_{H_{\sigma}}\right| \cap\left|\mathcal{A}_{M}\right|=$ $\left|\mathcal{A}_{H_{\sigma} \cap M}\right|$ are $\left|\mathcal{A}_{C}\right|$ where $C$ ranges over the components of $H_{\sigma} \cap M$. We next show for each $C$ the following properties (1)-(5):

(1) $\operatorname{st}\left(\mathcal{A}_{C} ; \mathcal{A}_{M}\right) \subseteq \operatorname{st}\left(\mathcal{A}_{C} ; \mathcal{A}_{N(C)}\right)$ are combinatorial balls of dimensions $(n-1)$ and $n$ respectively.

(2) $\operatorname{st}\left(\mathcal{A}_{C} ; \mathcal{A}_{M}\right) \cap \partial \operatorname{st}\left(\mathcal{A}_{C} ; \mathcal{A}_{N(C)}\right)=\partial \operatorname{st}\left(\mathcal{A}_{C} ; \mathcal{A}_{M}\right)$.

(3) $\operatorname{st}\left(\mathcal{A}_{C} ; \mathcal{A}_{M}\right)=\operatorname{st}\left(\mathcal{A}_{C} ; \mathcal{A}_{N(C)}\right) \cap \mathcal{A}_{M}$.

(4) The segment $\mathcal{A}_{C}$ is contained in the interior of the segment $\operatorname{st}\left(\mathcal{A}_{C} ; \mathcal{A}_{N(C)}\right) \cap \mathcal{A}_{H_{\sigma}}$.

(5) The balls $\left|\operatorname{st}\left(\mathcal{A}_{C} ; \mathcal{A}_{N(C)}\right)\right|$ and $\left|\operatorname{st}\left(\mathcal{A}_{D} ; \mathcal{A}_{N(D)}\right)\right|$ have disjoint interior whenever $D \neq$ $C$.

Properties (1)-(3) show that each pair $\left(\left|\operatorname{st}\left(\mathcal{A}_{C} ; \mathcal{A}_{N(C)}\right)\right|,\left|\operatorname{st}\left(\mathcal{A}_{C} ; \mathcal{A}_{O}\right)\right|\right)$ is a relative ball in $\left(\mathbb{R}^{n},\left|\mathcal{A}_{M}\right|\right)$; while properties (4) and (5) show that the family of such pairs is an admissible family or relative balls. Next we check properties (1)-(5) above. So, property (1) follows from Corollary 4.5 and Lemma 4.4. Also Lemma 4.4 yields properties (2) and (3). Moreover the components of $H_{\sigma} \cap M$ are pairwise disjoint digital segments and so property (5) is a direct consequence of Corollary 4.5. Finally we prove property (4). For this we consider the predecessor $\pi_{C}$ and the successor $\sigma_{C}$ of $C$. Since $\sigma \notin M$, Lemma 3.6 implies that neither $\pi_{C}$ nor $\sigma_{C}$ lie in $M$. Moreover one readily checks that, for $C=S_{\tau_{0}}^{k}=\left\{\tau_{0}, \tau_{1}, \ldots, \tau_{k}\right\}, L_{C}=\operatorname{st}\left(\mathcal{A}_{C} ; \mathcal{A}_{N(C)}\right) \cap \mathcal{A}_{H_{\sigma}}=\operatorname{st}\left(\mathcal{A}_{C} ; \mathcal{A}_{H_{\sigma}}\right)$ is the (closed) segment in $\mathbb{R}^{n}$ joining $c\left(\pi_{C} \cap \tau_{0}\right)$ to $c\left(\sigma_{C} \cap \tau_{k}\right)$. Here we use Lemma 4.4 with $O$ the digital line defined by $H_{\sigma}$. Furthermore $\mathcal{A}_{C}$ is the segment in $\mathbb{R}^{n}$ joining $c\left(\tau_{0}\right)$ to $c\left(\tau_{k}\right)$ and hence it is part of the interior of $L_{C}$. The proof of 4.1 is now finished.

In addition to Proposition 4.1 we also use the following two technical lemmas in the proof of Theorem 3.8 .

Lemma 4.6 Let $C$ be a component of $H_{\sigma} \cap M$. Then

$$
\left|\operatorname{st}\left(\mathcal{A}_{C} ; \mathcal{A}_{N(C)}\right)\right|-\left|\operatorname{st}\left(\mathcal{A}_{C} ; \mathcal{A}_{M}\right)\right| \subseteq\left|\mathcal{A}_{N(C) \cup M}\right|-\left|\mathcal{A}_{M}\right|
$$

Proof. Let $z \in \gamma-\partial \gamma$ with $\gamma \in \operatorname{st}\left(\mathcal{A}_{C} ; \mathcal{A}_{N(C)}\right)$ and $\gamma \notin \operatorname{st}\left(\mathcal{A}_{C} ; \mathcal{A}_{M}\right)$. Then $\gamma \in \mathcal{A}_{N(C) \cup M}$ by Lemma 4.2 and $\gamma \notin \mathcal{A}_{M}$ since $\operatorname{st}\left(\mathcal{A}_{C} ; \mathcal{A}_{M}\right)=\operatorname{st}\left(\mathcal{A}_{C} ; \mathcal{A}_{N(C)}\right) \cap \mathcal{A}_{M}$ by Lemma 4.4

Lemma 4.7 Let $(K, f)$ be a digital space and let $O_{1}$ y $O_{2}$ be two disjoint digital objects in $(K, f)$. Then each $\mathrm{O}_{2}$-component of $\mathrm{O}_{1}$ is contained in an $\mathrm{O}_{2}$-component of cell $_{n}(\mathrm{~K})-\mathrm{O}_{2}$.

Proof. Let $C$ be an $O_{2}$-component of $O_{1}$. For any $\sigma \in C$ let $C_{\sigma}$ denote the $O_{2}$-component of $\sigma$ in $\operatorname{cell}_{n}(K)-O_{2}$. Given $\tau \in C$ there exists an $O_{2}$-path $\left\{\sigma_{i}\right\}_{i=0}^{m}$ in $O_{1}$ with $\sigma_{0}=$ $\sigma$ and $\sigma_{m}=\tau$. Hence, for $1 \leq i \leq m$, there exist faces $\alpha_{i} \leq \sigma_{i-1} \cap \sigma_{i}$ for which $f\left(O_{1} \cup O_{2}, \alpha_{i}\right)=1$ and $f\left(O_{2}, \alpha_{i}\right)=0$. From property (3) in the definition of w.l.f.'s we obtain $f\left(\operatorname{cell}_{n}(K), \alpha_{i}\right)=1(1 \leq i \leq m)$. Thus $\left\{\sigma_{i}\right\}_{i=0}^{m}$ is also an $O_{2}$-path in $\operatorname{cell}_{n}(K)-O_{2}$, and so $\tau \in C_{\sigma}$. This shows $C \subseteq C_{\sigma}$. 

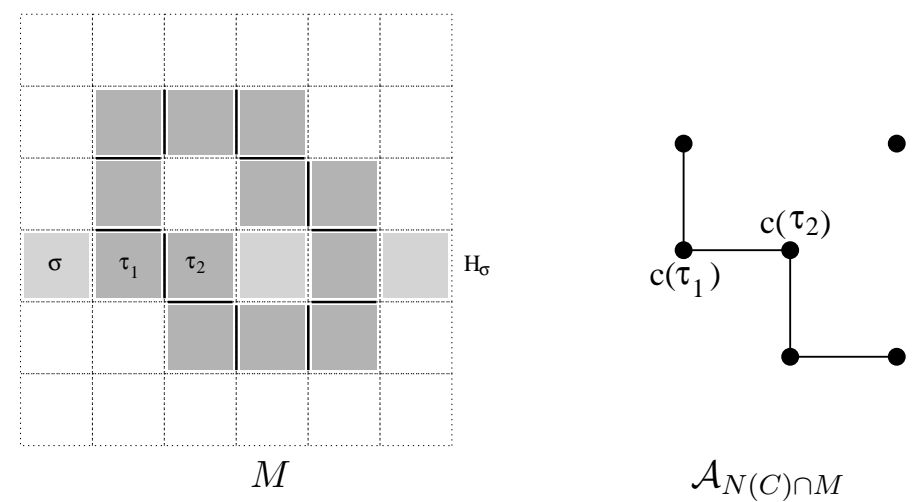

$\mathcal{A}_{N(C) \cap M}$

Fig. 3.

Proof of Theorem 3.8. The Digital Jordan-Brouwer Theorem (Th. 2.3) shows that the $M$ components of $\operatorname{cell}_{n}\left(R^{n}\right)-M$ are determined by the components of $\mathbb{R}^{n}-\left|\mathcal{A}_{M}\right|$. Hence it will suffice to check the equality

$$
i_{\text {dig }}(\sigma ; M)=i_{\text {top }}\left(c(\sigma) ;\left|\mathcal{A}_{M}\right|\right)
$$

To do that we first observe that $C$ is a component of $H_{\sigma} \cap M$ if and only if $\left|\mathcal{A}_{C}\right|$ is a component of $\left|\mathcal{A}_{H_{\sigma} \cap M}\right|=\left|\mathcal{A}_{H_{\sigma}}\right| \cap\left|\mathcal{A}_{M}\right|$. Here we use Lemma 3.6. Therefore it will be enough to show the equality

$$
i_{\text {dig }}(C ; M)=i_{\text {top }}\left(\left|\mathcal{A}_{C}\right| ;\left|\mathcal{A}_{M}\right|\right)
$$

for any component $C=S_{\tau_{0}}^{k}=\left\{\tau_{0}, \tau_{1}, \ldots, \tau_{k}\right\}$ of $H_{\sigma} \cap M$. For this let $\pi_{C}$ and $\sigma_{C}$ be the predecessor and the successor of $C$ respectively. It is readily checked that the centroids $c\left(\gamma_{0}\right)$ and $c\left(\gamma_{k}\right)$ of the $(n-1)$-faces $\gamma_{0}=\pi_{C} \cap \tau_{0}$ and $\gamma_{k}=\sigma_{C} \cap \tau_{k}$ respectively are separated by $\left|\mathcal{A}_{C}\right|$ in $\left|\operatorname{st}\left(\mathcal{A}_{C} ; \mathcal{A}_{N(C)}\right)\right| \cap\left|\mathcal{A}_{H_{\sigma}}\right|$. Moreover, by Proposition 4.1 we can use the relative ball $\left(\left|\operatorname{st}\left(\mathcal{A}_{C} ; \mathcal{A}_{N(C)}\right)\right|,\left|\operatorname{st}\left(\mathcal{A}_{C} ; \mathcal{A}_{M}\right)\right|\right)$ to compute the topological index in (**); and hence the equality will follow if we prove that both $c\left(\gamma_{0}\right)$ and $c\left(\gamma_{k}\right)$ belong to the same component of $D_{C}=\left|\operatorname{st}\left(\mathcal{A}_{C} ; \mathcal{A}_{N(C)}\right)\right|-\left|\mathcal{A}_{M}\right|$ if and only if both $\pi_{C}$ and $\sigma_{C}$ belong to the same $M$-component of $N(C)-M$. We next show this fact.

Assume $c\left(\gamma_{0}\right)$ and $c\left(\gamma_{k}\right)$ share the same component in $D_{C}$. Then, by Lemma 4.6, they are in the same component of $D_{C}^{\prime}=\left|\mathcal{A}_{N(C) \cup M}\right|-\left|\mathcal{A}_{M}\right|$, and hence the segments $L_{0}=\left\langle c\left(\gamma_{0}\right), c\left(\pi_{C}\right)\right\rangle$ and $L_{1}=\left\langle c\left(\gamma_{k}\right), c\left(\sigma_{C}\right)\right\rangle$ in $\mathcal{A}_{N(C) \cup M}-\mathcal{A}_{M}$ show that $c\left(\pi_{C}\right)$ and $c\left(\sigma_{C}\right)$ are both in the same component of $D_{C}^{\prime}$; therefore $\pi_{C}$ and $\sigma_{C}$ are in the same $M$-component of $N(C)-M=N(C) \cup M-M$ by Theorem 2.2. Conversely, if both $\pi_{C}$ and $\sigma_{C}$ lie in a $M$-component of $N(C)-M$ they are in the same $M$-component of $\operatorname{cell}_{n}\left(R^{n}\right)-M$ by Lemma 4.7; that is, $c\left(\gamma_{0}\right)$ and $c\left(\gamma_{k}\right)$ belong to the same component of $\mathbb{R}^{n}-\left|\mathcal{A}_{M}\right|$. Here we use again Theorem 2.2 and the segments $L_{0}$ and $L_{1}$ above. Moreover, by Proposition 4.1 $\left(\left|\operatorname{st}\left(\mathcal{A}_{C} ; \mathcal{A}_{N(C)}\right)\right|,\left|\operatorname{st}\left(\mathcal{A}_{C} ; \mathcal{A}_{M}\right)\right|\right)$ is a relative ball in $\left(\mathbb{R}^{n},\left|\mathcal{A}_{M}\right|\right)$, and hence $c\left(\gamma_{0}\right)$ and $c\left(\gamma_{k}\right)$ lie in the same component of $D_{C}$. The proof is now complete.

Remark 4.8 This proof generalizes to arbitrary solid digital spaces our Digital Index Theorem proved in [2, Th. 4] only for the digital space $\left(R^{n}, f_{\max }\right)$, where $f_{\max }$ is the lighting function given in Example 2.1(a). The proof of Theorem 4 in [2] uses, instead of Proposition 4.1, the fact that in $\left(R^{n}, f_{\max }\right)$ the set of pairs $\left(\left|\mathcal{A}_{N(C)}\right|,\left|\mathcal{A}_{N(C) \cap M}\right|\right)$ is an 
admissible family of relative balls, where $C$ ranges over the set of components of $H_{\sigma} \cap M$. This property is not longer true for arbitrary solid spaces, as it is shown in Fig. 3. In that figure we sketch a digital 1-manifold $M$ in the solid digital space $\left(R^{2}, g\right)$, with $g$ the w.l.f. given in Example 2.1(b), and the continuous analogue $\left|\mathcal{A}_{N(C) \cap M}\right|$ which is not an 1-ball.

\section{A Appendix: A topological index theorem}

It is part of the folklore concerning the Jordan-Brouwer Theorem that the component of a point $x$ in the complement of an $(n-1)$-manifold $M \subseteq \mathbb{R}^{n}$ is characterized by the number of points in $L \cap M$, where $L$ is a half-line emanating from $x$ which intersects $M$ transversally. However, it is not easy to find in literature a general and precise proof of this fact (see, for example, chapter 9 in [7] for one of these proofs). We state here a topological index theorem which is very convenient in dealing with digital topology. The details and proofs can be found in [2].

Let $M$ be a closed (i.e. compact and without boundary) connected combinatorial ( $n-1)$-manifold $M \subseteq \mathbb{R}^{n}$. We have the following well-known separation theorem.

Theorem A.1 (see [8, VIII.4.8]) If $M \subseteq \mathbb{R}^{n}$ is a closed $(n-1)$-manifold without boundary then $\mathbb{R}^{n}-M$ has two components, one bounded $B$, and one unbounded $U$. Moreover $M$ is the common boundary of both components.

In order to characterize the components of $\mathbb{R}^{n}-M$ we use relative balls. Namely, a relative ball in $\left(\mathbb{R}^{n}, M\right)$ is a pair of balls $\left(B^{n}, B^{n-1}\right)$ in $\mathbb{R}^{n}$ such that $B^{n-1} \subseteq B^{n}$, $B^{n-1} \cap \partial B^{n}=\partial B^{n-1}$ and $B^{n-1}=B^{n} \cap M$. It is not hard to show that any relative ball $\left(B^{n}, B^{n-1}\right)$ verifies that $B^{n}-B^{n-1}$ has exactly two components each of which is contained in a distinct component of $\mathbb{R}^{n}-M$. Given point $x \in \mathbb{R}^{n}-M$, let $\mathcal{S}_{x} \subseteq \mathbb{R}^{n}$ be an arbitrary half-line starting at $x$. As we work with polyhedral embeddings, it is easily checked that the intersection $\mathcal{S}_{x} \cap M$ consists of a union of a (possibly empty) finite family of pairwise disjoint arcs and points. Let $\mathcal{B}(x)=\left\{\left(B_{C}^{n}, B_{C}^{n-1}\right)\right\}$ be a family of relative balls where $C$ ranges over the set of components of $\mathcal{S}_{x} \cap M$. We say that $\mathcal{B}(x)$ is an admissible family of relative balls for $\mathcal{S}_{x} \cap M$ if $B_{C}^{n} \cap \mathcal{S}_{x}$ is an arc containing $C$ in its interior and int $B_{C}^{n} \cap$ int $B_{D}^{n}=\emptyset$ for all $C, D \subseteq \mathcal{S}_{x} \cap M$. The existence of such family $\mathcal{B}(x)$ of relative balls is an immediate consequence of the regular neighbourhood theory in pl-topology. By the use of an admissible family of relative balls we define the topological index of a point in the complement of the combinatorial manifold $M$ as follows.

Definition A.2 The (topological) index of a point $x \in \mathbb{R}^{n}-M$ is

$$
i_{\mathrm{top}}(x ; M)=\sum_{C} i_{\mathrm{top}}(C ; M),
$$

where $C$ ranges over the set of components of $\mathcal{S}_{x} \cap M$ and $i_{\text {top }}(C ; M)$ is defined as follows. Let $\mathcal{B}(x)=\left\{\left(B_{C}^{n}, B_{C}^{n-1}\right)\right\}$ be an admissible family of relative balls for $\mathcal{S}_{x} \cap M$. We write $i_{\text {top }}(C ; M)=0$ and say that $\mathcal{S}_{x}$ is tangent to $M$ at $C$ if the difference $B_{C}^{n} \cap \mathcal{S}_{x}-C$ (which is the union of two disjoint segments) is contained in one component of $B_{C}^{n}-B_{C}^{n-1}$. Otherwise we say that $\mathcal{S}_{x}$ is transversal to $M$ at $C$ and we write $i_{\text {top }}(C ; M)=1$.

It can be proved that the definition of $i_{\text {top }}(x ; M)$ does not depend on the choice of $\mathcal{B}(x)$. Moreover the topological index characterizes the components of $\mathbb{R}^{n}-M$ as follows. 
Theorem A.3 The point $x \in \mathbb{R}^{n}-M$ lies in the bounded component $B$ if and only if $i_{\text {top }}(x ; M)$ is odd.

Acknowledgements: This work has been partially supported by the projects DGICYT PB96-1374 and DGES PB96-0098C04-01.

\section{References}

[1] R. Ayala, E. Domínguez, A.R. Francés, A. Quintero, J. Rubio. On surfaces in digital topology. Proc. of the 5th Workshop on Discrete Geometry for Computer Imagery DGCI'95. (1995) 271-276.

[2] R. Ayala, E. Domínguez, A.R. Francés, A. Quintero. Determining the components of the complement of a digital $(n-1)$-manifold in $\mathbb{Z}^{n}$. Lecture Notes in Computer Science. 1176(1996) 163-176.

[3] R. Ayala, E. Domínguez, A.R. Francés, A. Quintero. Digital Lighting Functions. Lecture Notes in Computer Science. 1347(1997) 139-150.

[4] R. Ayala, E. Domínguez, A.R. Francés, A. Quintero. A Lighting Function for Strong 26-surfaces. Lecture Notes in Computer Science. 1568(1999) 91-103.

[5] R. Ayala, E. Domínguez, A.R. Francés, A. Quintero. Weak lighting functions and strong 26-surfaces. To appear in Theoretical Computer Science.

[6] G. Bertrand, R. Malgouyres. Some topological properties of discrete surfaces. Journal of Mathematical Imaging and Vision. 11(1999) 207-221.

[7] R.H. Bing. The geometric topology of 3-manifolds. Coll. Pub., 40. Amer. Math. Soc. 1983.

[8] A. Dold. Algebraic Topology. Die Grundlehren der Math., 200. Springer, 1972.

[9] T.Y. Kong, A.W. Roscoe. Continuous Analogs of Axiomatized Digital Surfaces. Computer Vision, Graphics, and Image Processing. 29(1985) 60-86.

[10] R. Kopperman, P.R. Meyer, R.G. Wilson. A Jordan Surface Theorem for threedimensional digital spaces. Discrete and Computational Geometry. 1991. 155-161.

[11] V.A. Kovalevsky. Finite topology as applied to image analysis. Computer Vision, Graphics, and Image Processing. 46(1989) 141-161.

[12] R. Malgouyres. A definition of surfaces in $\mathbb{Z}^{3}$ : a new 3D discrete Jordan Theorem. Theoretical Computer Science. 186 (1997) 1-41.

[13] D.G. Morgenthaler, A. Rosenfeld. Surfaces in Three-Dimensional Digital Images. Information and Control. 51(1981) 227-247.

[14] F. Raymond. Separation and union theorems for generalized manifolds with boundary. Michigan Math. J. 7(1970) 1-21.

[15] C.P. Rourke, B.J. Sanderson. Introduction to Piecewise linear topology. Ergebnisse der Math., 69. Springer, 1972. 